\title{
Role of the Primary Care Physician in the Diagnosis and Management of Hepatitis C Virus Infection
}

David H. Spach, $M D$

In the past several years, hepatitis $C$ virus (HCV) has firmly captured the attention of medical providers, the lay public, and governmental health care agencies. Indeed, with an estimated prevalence of 3.9 million persons infected with $\mathrm{HCV}$ in the United States (and 2.7 million of these with chronic infection), ${ }^{1}$ this viral disease could become the most important infectious disease in this country during the next decade. Given the emerging clinical importance of $\mathrm{HCV}$ infection, the findings of Nicklin and colleagues, presented in this issue of the Fournal of the American Board of Family Practice, ${ }^{2}$ should raise some eyebrows and generate controversy regarding the role the primary care provider should play in the diagnosis and treatment of persons infected with $\mathrm{HCV}$.

The current study was conducted in November 1997, and the final analysis involved 172 community-based internists and family physicians employed in the greater metropolitan Philadelphia area. The investigators used a survey to gather information regarding the current practices of these primary care physicians for diagnosing and treating $\mathrm{HCV}$ infection. Seventy percent of the physicians routinely tested alanine transaminase (ALT) levels as part of their complete checkup, and, if the ALT level was elevated, $64 \%$ then obtained HCV antibody testing. Once the diagnosis of $\mathrm{HCV}$ infection was made, $62 \%$ advised using condoms in a monogamous relationship, and $56 \%$ recommended abstaining from alcohol, with $44 \%$ considering limited alcohol consumption acceptable. If the $\mathrm{HCV}$-infected person had an elevated ALT, $82 \%$ recommended liver biopsy and $77 \%$ recommended interferon- $\alpha$ therapy. A selected

\footnotetext{
Submitted 2 August 1999.
}

From the Division of Infectious Diseases, Department of Medicine, University of Washington, Seattle. Address reprint requests to David $\mathrm{H}$. Spach, $\mathrm{MD}$, Harborview Medical Center, Mailstop 359930, 3259 th Ave, Seattle, WA 981042499. chart audit, however, estimated only $36 \%$ of patients actually underwent liver biopsy and only $29 \%$ had documented interferon- $\alpha$ therapy. Fortynine percent routinely referred $\mathrm{HCV}$-infected patients to a specialist, with $51 \%$ referring only some patients. At first glance, some of these findings might appear to show that the primary care providers did not diagnose and manage $\mathrm{HCV}$ infection adequately.

The authors reported that approximately one third of the physicians in this study did not perform $\mathrm{HCV}$ antibody testing in patients who had elevated ALT levels. In the National Institutes of Health (NIH) Consensus Development Conference, the panel recommends, "Individuals with mildly elevated ALT levels, with or without risk factors for hepatitis C, should be tested for antibody by EIA and, if positive, the results confirmed by either supplemental RIBA or qualitative HCV RNA by PCR." ${ }^{3}$ In addition, the Centers for Disease Control and Prevention (CDC) HCV guidelines recommend routine testing for $\mathrm{HCV}$ in persons with persistently abnormal alanine aminotransferase levels. ${ }^{4}$ Remember, however, that the NIH consensus guidelines were not published until September 1997 (only several months before this study was performed), and the guidelines appeared in the journal Hepatology, a journal certainly not routinely subscribed to or reviewed by primary care providers. Although the CDC guidelines were published in the widely circulated Morbidity and Mortality Weekly Report (MMWR), these guidelines did not appear until October 1998, almost 1 year after the physician survey was conducted. Regardless of what primary care providers should have known in November 1997, they should now have familiarity with the formal recommendations issued by the CDC that address indications for $\mathrm{HCV}$ testing (Table 1).

Sixty-two percent of the physicians interviewed in the current study advised patients to use a con- 
Persons who should be tested routinely for hepatitis $\mathrm{C}$ virus (HCV) infection based on their risk for infection or based on a recognized exposure
Persons for whom routine $\mathrm{HCV}$ testing is not recommended

Persons for whom routine HDV testing is of uncertain need
1. Persons who ever injected illegal drugs, including those who injected once or a few times many years ago and do not consider themselves to be drug users

2. Persons with selected medical conditions, including persons who received clotting factor concentrates produced before 1987 , persons who were ever on chronic (long-term) hemodialysis, and persons with persistently abnormal alanine aminotransferase levels

3. Recipients of transfusions or organ transplants, including persons who were notified that they received blood from a donor who later tested positive for $\mathrm{HCV}$ infection, persons who received a transfusion of blood or blood components before July 1992, and persons who received an organ transplant before July 1992

4. Health care, emergency medical, and public safety workers after needle sticks, sharps, or mucosal exposures to $\mathrm{HCV}$-positive blood

5. Children born to HCV-positive women

1. Health care, emergency medical, and public safety workers

2. Pregnant women

3. Household (nonsexual) contacts of HCV-positive persons

4. The general population

1. Recipients of transplanted tissue (eg, corneal, musculoskeletal, skin, ova, sperm)

2. Intranasal cocaine and other noninjecting illegal drug users

3. Persons with a history of tattooing or body piercing

4. Persons with a history of multiple sex partners or sexually transmitted diseases

5. Long-term steady sex partners of HCV-positive persons

Modified from Centers for Disease Control and Prevention. Recommendations for prevention and control of hepatitis C virus (HCV) infection and HCV-related disease. MMWR Morb Mortal Wkly Rep 1998;47(RR-19):1-39. ${ }^{4}$

dom in a monogamous relationship. Available data suggest that sexual transmission of $\mathrm{HCV}$ accounts for at least $15 \%$ of all cases of $\mathrm{HCV}$ infection, ${ }^{5}$ with high-risk sexual behavior a clearly defined risk factor. ${ }^{1}$ Studies with long-term spouses of patients with chronic $\mathrm{HCV}$ infection, however, have shown a transmission rate of only about $1.5 \% .^{4}$ Indeed, the $\mathrm{CDC}$ recommends that $\mathrm{HCV}$-positive persons with long-term steady partners do not need to change their sexual practices. More specifically, the CDC recommendations state, "if the partner tests negative, the couple should be informed of available data regarding risk for $\mathrm{HCV}$ transmission by sexual activity to assist them in making decisions about precautions." ${ }^{4}$ Thus, the recommendation for $\mathrm{HCV}$-infected persons to use condoms in a monogamous relationship is far from dogmatic. For those persons with ongoing high-risk sexual activity involving multiple partners, however, the use of condoms is recommended, not only to prevent $\mathrm{HCV}$, but to prevent acquisition and transmission of other important sexually transmitted diseases.

Several studies have suggested that chronic, heavy alcohol use in HCV-infected persons accel- erates the course of $\mathrm{HCV}$-related liver disease. In the present study, $56 \%$ of the physicians recommended $\mathrm{HCV}$-infected patients should abstain from alcohol, with $44 \%$ considering limited alcohol consumption acceptable. The NIH consensus guidelines recommend that patients with chronic hepatitis $\mathrm{C}$ should ingest less than $10 \mathrm{~g} / \mathrm{d}$ of alcohol, but those who have cirrhosis or are planning to receive interferon- $\alpha$ therapy should be encouraged to abstain from alcohol. ${ }^{6}$ The cutoff of $10 \mathrm{~g} / \mathrm{d}$ arose from one study that showed liver function tests and HCV RNA levels did not change in persons who ingested low amounts of alcohol $(<10 \mathrm{~g} / \mathrm{d}){ }^{7}$

When evaluating $\mathrm{HCV}$-infected patients who had an elevated ALT levels, $82 \%$ of the physicians in the present study recommended liver biopsy and $77 \%$ of the physicians recommended interferon- $\alpha$ therapy. When the investigators performed a selected chart audit, they found only $36 \%$ of patients actually underwent liver biopsy and only $29 \%$ received interferon therapy. The NIH consensus guidelines recommend liver biopsy in $\mathrm{HCV}$-infected persons to (1) predict the likelihood of responding to therapy, (2) to determine the staging of the liver disease, and (3) to rule out an unsuspected 
secondary hepatic disease. ${ }^{3}$ Considering the cost of the biopsy ( $\$ 1500$ to $\$ 2000$ ) and a death rate of 1 in 10,000 liver biopsies, some have questioned the routine need for biopsy and have argued that liver biopsy serves as a barrier for a considerable number of patients interested in therapy. ${ }^{8}$ Can noninvasive measures provide the same information that liver biopsy does? Unfortunately, available data suggest that HCV RNA levels do not clearly correlate with liver histology. Moreover, although increased ALT levels suggest active liver disease, a normal ALT reading does not rule out abnormal liver histology.

Whether all HCV-infected patients should undergo liver biopsy before treatment is now a hotly contested issue. From a clinical standpoint, liver biopsy is currently used predominantly to determine whether a patient has a histologic indication for HCV therapy. Accordingly, clinicians are unlikely to pursue biopsy in patients who are not good candidates for interferon- $\alpha$ therapy, such as those with cardiovascular disease, psychiatric disease, or ongoing abundant ingestion of alcohol.

In 1997, therapy for HCV consisted of interferon- $\alpha$ therapy alone, with long-term response rates of less than $20 \%$. Given the serious adverse effects and low response rates to interferon- $\alpha$, many providers in the United States questioned the overall benefit of $\mathrm{HCV}$ treatment-in essence, was the juice worth the squeeze? The enthusiasm for treating HCV changed dramatically approximately 1 year ago with data that showed ribavirin added to interferon- $\alpha$ is clearly superior to interferon- $\alpha$ therapy alone, with an approximate doubling in the percentage of persons who have long-term response rates. ${ }^{9}$ Subsequent studies have shown similar data. ${ }^{10}$ Further enthusiasm arose with data that showed interferon- $\alpha$ therapy decreases the risk of hepatocellular carcinoma in persons with chronic $\mathrm{HCV}$ who have a sustained response to therapy. ${ }^{11}$

So, overall, how would I judge the performance of the primary care providers in this study? By 1999 standards, not so good. But, by 1997 standards, which is when the study was conducted, their performance is hard to criticize. Regardless of how one interprets these findings, the study by Nicklin and co-workers should generate enhanced interest and understanding of several specific recommendations for the care of $\mathrm{HCV}$-infected persons.

On a larger scale, this interesting study raises several critical issues related to health care delivery. First, who should take care of patients with chronic
HCV infection? In this study, $49 \%$ of the physicians routinely referred these patients to specialists. From an ideal standpoint, $\mathrm{HCV}$-infected patients would probably receive optimal care if they could see a specialist. If so, then, what is the role of the primary care provider for this disease? From my perspective, all primary care providers, at the least, should have a clear understanding of who and how to test for HCV infection. After all, because primary care providers serve on the front line of medical care, they are the most likely to have the first encounter with a person who does not know they are infected with $\mathrm{HCV}$. As the number of chronically $\mathrm{HCV}$-infected persons enter into the medical community, however, they will surely overwhelm the capacity of the specialists, and some primary care providers will need to also play a role in treatment, especially in managed care settings and in more rural areas where fewer specialists practice.

Looking back at the earlier years of the HIV epidemic, a similar situation occurred when the number of HIV-infected persons overwhelmed the capacity of specialists, and many primary care providers were thrust into managing complex patient problems in a very rapidly changing field. A study published in 1996 reported that HIV-infected persons cared for by physicians with greater HIV experience had markedly improved survival rates when compared with those cared for by physicians with less HIV experience; this study was performed in a large managed care setting, and all physicians in this study were primary care providers. ${ }^{12}$ Many have misinterpreted this study to mean that specialists provide better HIV care than nonspecialists, but what the study really showed was that experience matters, probably because the experienced providers had enhanced knowledge about HIV.

Extrapolating from this study, I believe physician experience and expertise will affect the outcomes of persons with HCV. Accordingly, those primary care providers interested in managing $\mathrm{HCV}$ infection will need to have a particular interest in this area, make a serious commitment to remaining current in this field, and should gain experience while having back-up from a specialist in this field. Innovative mentoring programs could be developed to allow primary care physician to gather experience while working with a specialist; this type of system could lead to a source of longterm expert back-up. 
This study also raises issues related to the dissemination of medical information in a rapidly changing field, such as $\mathrm{HCV}$. The challenge is to translate the massive amount of new information into practical terms, disseminate it on a timely basis, and then have this information become incorporated by clinicians. Often a breakdown occurs at each of these steps. The new information is typically generated by experts in the field, but many experts often forget the extensive demand placed on a primary care physician to keep up with a vast array of important clinical diseases. The primary care physician needs to have clear-cut, succinct, regularly updated guidelines that are disseminated in widely read medical journals and available electronically. In the HIV field, the CDC has generated excellent, regularly updated guidelines on the prevention of opportunistic infections and, similarly, the NIH has recently sponsored excellent antiretroviral therapy guidelines. In the future, I would hope that the NIH and the CDC would envision sponsoring regularly updated $\mathrm{HCV}$ guidelines that clearly advise specialists and primary care physicians how best to screen for, diagnose, and treat $\mathrm{HCV}$ infection.

\section{References}

1. Alter MJ, Kruszon-Moran D, Nainan OV, et al. The prevalence of hepatitis $C$ virus infection in the United States, 1988 through 1994. N Engl J Med 1999;341:556-62.

2. Nicklin DE, Schultz C, Brensinger C, et al. Current care of hepatitis $C$ positive patients by primary care physicians in an integrated delivery system. J Am Board Fam Pract 1999;12:427-35.

3. National Institutes of Health Consensus Development Conference Panel statement: management of hepatitis C. Hepatology 1997;26 (3 Suppl 1):2S-10S. (Also available electronically from http://consensus.nih.gov).

4. Recommendations for prevention and control of hepatitis $\mathrm{C}$ virus (HCV) infection and $\mathrm{HCV}$-related chronic disease. Centers for Disease Control and Prevention. MMWR Morb Mortal Wkly Rep 1998; 47(RR-19):1-39.

5. Alter MJ. Epidemiology of hepatitis C. Hepatology 1997;26(3 Suppl 1):62S-65S.

6. Schiff ER. Hepatitis C and alcohol. Hepatology 1997;26(3 Suppl 1):39S-42S.

7. Cromie SL, Jenkins PJ, Bowden DS, Dudley FJ. Chronic hepatitis C: effect of alcohol on hepatic activity and viral titre. J Hepatol 1996;25:821-6.

8. Wong JB, Bennett WG, Koff RS, Pauker SG. Pretreatment evaluation of chronic hepatitis $\mathrm{C}$ : risks, benefits, and costs. JAMA 1998;280:2088-93.

9. Poynard T, Marcellin P, Lee SS, et al. Randomised trial of interferon alpha $2 \mathrm{~b}$ plus ribavirin for 48 weeks or for 24 weeks versus interferon alpha2b plus placebo for $\mathbf{4 8}$ weeks for treatment of chronic infection with hepatitis $\mathrm{C}$ virus. International Hepatitis Interventional Therapy Group. Lancet 1998;352:142632.

10. McHutchison JG, Gordon SC, Schiff ER, et al. Interferon alpha- $2 \mathrm{~b}$ alone or in combination with ribavirin as initial treatment for chronic hepatitis $C$. Hepatitis Interventional Therapy Group. N Engl J Med 1998;339:1485-92.

11. Imai $Y$, Kawata $S$, Tamura $S$, et al. Relation of interferon therapy and hepatocellular carcinoma in patients with chronic hepatitis C. Osaka Hepatocellular Carcinoma Prevention Study Group. Ann Intern Med 1998;129:94-9.

12. Kitahata MM, Koepsell TD, Deyo RA, Maxwell CL, Dodge WT, Wagner EH. Physicians' experience with the acquired immunodeficiency syndrome as a factor in patients' survival. N Engl J Med 1996;334: 701-6. 